Published in final edited form as:

Atherosclerosis. 2018 February ; 269: 86-91. doi:10.1016/j.atherosclerosis.2017.12.026.

\title{
The relationship of circulating fibroblast growth factor 21 levels with incident atrial fibrillation: The Multi-Ethnic Study of Atherosclerosis
}

\author{
Tsz Him Hui ${ }^{a}$, Robyn L. McClelland ${ }^{\mathrm{b}}$, Matthew A. Allison ${ }^{\mathrm{c}}$, Carlos J. Rodriguez ${ }^{\mathrm{d}, \mathrm{e}}$, Richard \\ A. Kronmal ${ }^{b}$, Susan R. Heckbert ${ }^{f}$, Erin D. Michos ${ }^{g, h}$, Philip J. Barter ${ }^{a}$, Kerry-Anne Rye ${ }^{a}$, and \\ Kwok Leung Onga, ${ }^{\mathrm{*}}$ \\ aLipid Research Group, School of Medical Sciences, University of New South Wales, Sydney, \\ NSW, Australia \\ bDepartment of Biostatistics, University of Washington, Seattle, WA, USA \\ 'Department of Family Medicine and Public Health, University of California San Diego, La Jolla, \\ CA, USA \\ dDepartment of Epidemiology and Prevention, Wake Forest University School of Medicine, \\ Winston-Salem, NC, USA \\ eDepartment of Medicine, Wake Forest University School of Medicine, Winston Salem, NC, USA \\ fDepartment of Epidemiology, Cardiovascular Health Research Unit, University of Washington, \\ Seattle, WA, USA \\ 9Department of Epidemiology, Johns Hopkins University Bloomberg School of Public Health, \\ Baltimore, MD, USA \\ hDivision of Cardiology, Johns Hopkins School of Medicine, Baltimore, MD, USA
}

\begin{abstract}
Background and aims-Elevated circulating levels of fibroblast growth factor 21 (FGF21) are associated with multiple cardiovascular disease (CVD) risk factors and incident events. Previous small cross-sectional studies, mainly in Chinese populations, have suggested FGF21 may play a role in the development of atrial fibrillation (AF). We therefore investigated the relationship of FGF21 levels with incident AF in participants free of clinically apparent CVD at baseline in a large, multi-ethnic cohort.
\end{abstract}

\footnotetext{
"Corresponding author: Kwok-Leung Ong, School of Medical Sciences, University of New South Wales, Sydney, NSW 2052, Australia, oklws@yahoo.com.hk.

Conflict of interest: The authors declared they do not have anything to disclose regarding conflict of interest with respect to this manuscript.

Author contributions: T.H.H. and K.L.O. participated in data analysis and wrote the manuscript; R.L.M. participated in data analysis M.A.A., K.A.R. and K.L.O. participated in the study design. C.J.R., R.A.K., S.R.H., E.D.M. and K.L.O. participated in acquisition of the data. All authors participated in data interpretation and critical revision of the manuscript.

Publisher's Disclaimer: This is a PDF file of an unedited manuscript that has been accepted for publication. As a service to our customers we are providing this early version of the manuscript. The manuscript will undergo copyediting, typesetting, and review of the resulting proof before it is published in its final citable form. Please note that during the production process errors may be discovered which could affect the content, and all legal disclaimers that apply to the journal pertain.
} 
Methods-A total of 5,729 participants of four major ethnic groups (Caucasian, African American, Hispanic American, and Chinese American) from the Multi-Ethnic Study of Atherosclerosis (MESA), who were free of AF and had plasma FGF21 levels measured by ELISA at the baseline exam, were included in the analysis. Participants were followed up for incident AF over a median period of 12.9 years. Cox proportional hazards regression analysis was used.

Results-Among the 5,729 participants, 778 participants developed incident AF. Participants with incident AF had significantly higher baseline FGF21 levels than those without incident AF (median $=166.0$ and $142.8 \mathrm{pg} / \mathrm{mL}, p<0.001)$. After adjusting for possible confounders, including demographic, socioeconomic and lifestyle factors, traditional CVD risk factors and circulating inflammatory markers, higher baseline FGF21 levels did not predict incident AF over the follow up period. There was no effect modification by sex or ethnicity.

Conclusions-Baseline FGF21 levels were not associated with the development of AF in an ethnically diverse population followed long-term. Our findings do not support an important role of FGF21 in AF development.

\section{Keywords}

atrial fibrillation; cardiovascular disease; fibroblast growth factor 21; Multi-Ethnic Study of Atherosclerosis

\section{Introduction}

Atrial fibrillation (AF) is a global prevalent health problem. Although it contributes to increased morbidity, mortality, and healthcare cost [1], it can be difficult to detect when paroxysmal [2]. Therefore, many studies have explored the potential of biomarkers to improve early AF risk screening, detection and disease management [3]. The most wellestablished AF biomarker is N-terminal pro B-type natriuretic peptide (NT-proBNP) elevated circulating levels of which are predictive of incident AF [4]. Recently, fibroblast growth factor 21 (FGF21) has been suggested as a potential biomarker for AF risk.

FGF21 influences glucose and lipid metabolism [5-7]. Moreover, FGF21 improves tissue sensitivity to insulin, lowers blood glucose levels and has been implicated as a potential biomarker for early detection of cardiometabolic disorders [8]. Its circulating levels are often elevated in obesity, dyslipidemia, insulin resistance, the metabolic syndrome, type 2 diabetes, non-alcoholic fatty liver disease and coronary artery disease [8]. Elevated FGF21 levels in this context may be due to the presence of FGF21 resistance, as a result of impaired FGF21 signaling or compensatory responses to the underlying metabolic stress, as FGF21 shows cardioprotective properties in cell culture and animal studies [8].

Recent studies in a Chinese population suggest an association between FGF21 and AF. In a cross-sectional study of $113 \mathrm{AF}$ patients and 60 healthy control subjects, serum FGF21 levels were found to be elevated in AF, and varied according to AF type [9]. Elevated FGF21 mRNA levels in atrial tissue have also been reported in AF patients [10]. However, the association of FGF21 levels with the incident development of AF, particularly among an ethnically diverse population, is unknown. Moreover, it is also unclear whether circulating FGF21 can predict AF development, independent of other CVD risk factors and more well- 
established biomarkers such as NT-proBNP, and cardiac magnetic resonance imaging (MRI) parameters. Given the potential relationship of FGF21 with AF, we conducted an analysis to test the hypothesis of a significant association between baseline FGF21 levels and incident AF in the Multi-Ethnic Study of Atherosclerosis (MESA). Ours is the first longitudinal analysis to investigate the relationship between baseline FGF21 and incident AF in a large well-established multi-ethnic cohort. This will provide insights on whether FGF21 may play a role in $\mathrm{AF}$ development and whether it could be used as a biomarker for predicting future risk of $\mathrm{AF}$.

\section{Patients and methods}

\subsection{Study participants}

The MESA is a longitudinal cohort of 6,814 men and women aged 45-84 years, and free of clinically apparent CVD at baseline [11]. At baseline, none of the participants reported a history of physician-diagnosed CVD, current AF, or had undergone procedures related to CVD. Participants of four major ethnic groups (Caucasian, African American, Hispanic American, and Chinese American) were recruited from six United States communities between July 2000 and August 2002. After the baseline exam, participants attended up to four additional clinic visits over a 10-year period. The study was approved by the institutional review boards at all participating centers and informed written consent was obtained from all participants. The study was performed in compliance with the principles of the Declaration of Helsinki. Details of the study objectives, design, and protocol have been described previously [11]. Among 6,814 participants at baseline, data on FGF21 levels were available on 5,792 participants. After further excluding 59 participants with either a history of AF at baseline or with AF detected by electrocardiogram at the baseline exam, and 4 participants with missing follow-up data for AF, a total of 5,729 participants were included in this analysis.

\subsection{Event ascertainment}

At intervals of 9-12 months, a trained telephone interviewer contacted each participant to inquire about all interim hospital admissions, cardiovascular outpatient diagnoses and procedures, and deaths. Additional medical encounters were identified through follow-up visits, participant call-ins, medical record abstractions, and obituaries. Copies of all death certificates and medical records for all hospitalizations, and selected outpatient cardiovascular diagnoses and procedures were requested to verify self-reported diagnoses. Out of hospital cardiovascular deaths were identified through next of kin interviews. Adjudicated coronary heart disease (CHD) end-points included myocardial infarction, resuscitated cardiac arrest, angina, and CHD death.

Incident AF was ascertained from ICD-9 (International Classification of Diseases, Ninth Revision) codes (427.31- AF and 427.32 - atrial flutter) assigned at hospital discharge $[12,13]$, an ICD-10 codes (I48 - AF and atrial flutter) on the death certificate, or based on the presence of AF detected by a 12-lead electrocardiogram conducted at the fifth study exam in 2010-2012. For participants older than 65 years of age who were enrolled in fee-for service Medicare, claims data for inpatient and outpatient services were also used to ascertain the 
diagnosis of AF. Follow-up started from the baseline examination until death, loss to followup, or 31 December 2014, whichever came first, with a median follow-up time of 12.9 years.

\subsection{Laboratory measurement}

Venous blood samples were collected after a 12-hour fast by certified technicians using standardized venipuncture procedures. FGF21 levels were measured from stored plasma samples obtained at the baseline exam using enzyme-linked immunosorbent assay kits from the Antibody and Immunoassay Services, University of Hong Kong, Hong Kong (www.antibody.hku.hk) as described previously [14,15]. Two in-house controls (high- and low-level controls) were run in each assay. The intra-assay and inter-assay coefficients of variation were $<10 \%$.

HDL cholesterol was measured using the cholesterol oxidase method (Roche Diagnostics, Indianapolis, IN) after precipitation of non-HDL cholesterol (non-HDL-C) with magnesium/ dextran sulphate. Triglyceride concentrations were measured using a glycerol-blanked enzymatic method with the Triglyceride GB reagent (Roche Diagnostics) on the Roche COBAS FARA centrifugal analyzer. In plasma samples with a triglyceride value $<400$ $\mathrm{mg} / \mathrm{dl}$, LDL cholesterol was calculated using the Friedewald formula. LDL cholesterol data were not available in plasma samples with a triglyceride value $\geq 400 \mathrm{mg} / \mathrm{dl}$ ( $\mathrm{n}=68$ out of 5,729 participants included in this analysis). High-sensitivity C-reactive protein (CRP), fibrinogen, and interleukin-6 (IL-6) levels were measured in all participants at the baseline exam as described previously [16]. Insulin resistance was estimated using the homeostasis model assessment index (HOMA2-IR), according to the updated computer model as described previously [17]. Serum creatinine was measured by rate reflectance spectrophotometry using thin film adaptation of the creatine amidinohydrolase method on the Vitros analyzer (Johnson \& Johnson Clinical Diagnostics Inc, Rochester, NY). Estimated glomerular filtration rate (eGFR) was calculated using the creatinine-based Chronic Kidney Disease Epidemiology Collaboration equation [18]. Baseline NT-proBNP levels were measured as described previously $[19,20]$.

\subsection{Other variables of interest}

Information on age, ethnicity, education, health insurance, smoking, alcohol use, total gross family income, physical activity, medical history and medication use were obtained using standardized questionnaires. Body mass index (BMI) was measured as the weight in kilograms divided by height in meters squared. A standard flexible tape measure was used to measure hip and waist circumferences. Resting blood pressure (BP) was measured three times in a seated position and the average of the last two BP readings was used in the analysis. Hypertension was defined as BP $\geq 140 / 90 \mathrm{mmHg}$, previous diagnosis of hypertension, or use of anti-hypertensive medications. Physical activity was measured as the total number of hours of self-reported moderate and vigorous activity per week, multiplied by metabolic equivalent (MET) level as described elsewhere [21]. Current alcohol use was categorized based on the largest number of drinks per day in the past month into no or moderate consumption (0-1 drinks/day for women and 0-2 drinks/day for men), high consumption (2-3 drinks/day for women and 3-4 drinks/day for men) and heavy drinking ( $\geq 4$ drinks/day for women and $\geq 5$ drinks/day for men) as described previously [22]. The 
cardiac MRI variables, including left ventricular (LV) mass, LV end-diastolic volume and ejection fraction, were measured as described previously [23].

\subsection{Statistical analysis}

Data are presented as mean (standard deviation) or percentage (number). For variables with a skewed distribution, data are presented as median (interquartile range). Comparison of baseline clinical characteristics between participants with and without incident AF was performed by independent chi-square test for categorical variables and $t$-test for continuous variables. For skewed variables, data were analyzed after natural $\log (\ln )$ transformation.

The association of baseline FGF21 levels (as a continuous variable) with incident AF events was assessed using Cox proportional hazards regression analysis and hazard ratios were estimated after adjusting for possible confounders. In model 1, the results were adjusted for demographic data, including age, sex, and ethnicity. In model 2, data were further adjusted for socioeconomic and lifestyle factors, including education, smoking, pack-years of smoking, current alcohol use, health insurance and physical activity. In model 3, data were further adjusted for traditional cardiovascular risk factors, including waist-to-hip ratio, height, heart rate, diabetes, systolic BP, HOMA-IR, HDL cholesterol, LDL cholesterol, triglycerides, eGFR, NT-proBNP, use of lipid-lowering medication, use of hypertensive medication, and use of aspirin. In model 4, data were further adjusted for circulating inflammatory markers, including CRP and IL-6. In model 5, data were further adjusted for baseline MRI measures of LV structure and function, including LV mass, LV end-diastolic volume, and LV ejection fraction. As plasma FGF21 levels and NT-proBNP levels were highly skewed, data were ln-transformed in the Cox regression analysis to prevent unstable estimates of effects since extreme values may have undue influence on the estimate of the regression coefficient. FGF21 in quartiles was also modeled in a separate analysis. Survivals were estimated by the Kaplan-Meier method and compared by the log-rank test. The proportional hazard assumption was checked by using Schoenfeld residuals. As reports have shown ethnic differences in AF prevalence, sex differences in FGF21 levels, and a close relationship of FGF21 levels with obesity, we also investigated whether there was an interaction of effect by sex, race/ethnicity (Caucasian, African American, Hispanic American, and Chinese American), and BMI ( $<25.0,25.0-29.0$, and $\left.330.0 \mathrm{~kg} / \mathrm{m}^{2}\right)$. In a separate analysis, a time-dependent Cox model was used to account for the possibility that incident CHD might have been a confounder. A two-tailed $p<0.05$ was considered statistically significant. Data analysis was performed using SPSS 24 (IBM, Armonk, NY) or STATA 14.0 (StataCorp, College Station, TX).

\section{Results}

Among the 5,729 participants, 778 participants developed incident AF over a median followup period of 12.9 years. Table 1 shows the baseline characteristics of the participants with and without incident AF. Compared to those who did not have incident AF, participants with incident AF were more likely to be older, male, and Caucasian, with higher pack-years of smoking, waist-to-hip ratio, height, prevalence of diabetes, systolic blood pressure, LV mass, IL-6 and NT-proBNP levels, and lower eGFR. They were less likely to be ever smokers, be 
physically active and have health insurance, and used anti-hypertensive medication and aspirin more often than those who did not have AF during follow-up. Although participants with incident AF had lower LDL cholesterol levels than those without AF, they were more likely to take lipid-lowering medications.

As shown in Table 1, plasma FGF21 levels at baseline were significantly higher in participants with incident AF, compared to those without incident AF. Fig. 1 shows the Kaplan-Meier cumulative curves for incident AF events over time across quartiles of FGF21 levels at baseline. Participants with higher quartile FGF21 levels at baseline had a higher risk of incident $\mathrm{AF}$ (log-rank test $p<0.001)$. However, as shown in Table 2, the association of ln-transformed FGF21 levels with incident AF was not significant after adjusting for possible confounders, including demographic, socioeconomic and lifestyle factors, traditional CVD risk factors, and circulating inflammatory markers ( $p=0.62$, model 4$)$. Further adjustment for LV mass, LV end-diastolic volume, and LV ejection fraction resulted in similar findings ( $p=0.59$, model 5). Similar results were also obtained when FGF21 levels were assessed as quartiles. In all these adjusted models, no significant interaction was found with sex, race/ethnicity and BMI groups (all $p>0.10$, Supplementary Tables 1-3).

In a separate analysis, we assessed the relationship of baseline FGF21 levels with incident AF events using time-dependent Cox regression model to further adjust for incident CHD (Table 3). However, including incident CHD as a time-dependent covariate did not change the findings for baseline FGF21 levels.

\section{Discussion}

With a sample size of 5,729 participants recruited from 6 communities across the United States, this is the largest study on the relationship of FGF21 levels and AF. In the previous reports, plasma FGF21 levels have been reported to be associated with CHD [24], carotid atherosclerosis [25], and different cardiovascular risk factors, such as obesity, metabolic syndrome, type 2 diabetes, hypertriglyceridemia, hyperinsulinemia, pericardial fat volume, hypertension, and renal function [8,26-30]. In our previous study in patients with type 2 diabetes, elevated FGF21 levels were associated with incident total CVD events [15]. In a previous small case-control study, FGF21 levels were reported to be elevated in AF, and differed significantly across different AF types [9]. However, we did not observe a significant association of FGF21 levels with incident AF in a multi-ethnic cohort of participants who were apparently free of baseline CVD in this study.

Previous basic science studies have suggested a potential role of FGF21 in cardiac function and pathogenesis of AF and other related CVD events. FGF21 is expressed and released by cardiomyocytes [31]. It protects against cardiac hypertrophy in mice, and prevents the production of reactive oxygen species in cardiac cells $[31,32]$. FGF21 can also ameliorate ischaemia/reperfusion injury in rat cardiomyocytes by inhibiting oxidative stress and inflammation, and improving energy supply [33]. An in vivo mouse study revealed that FGF21 secretion from hepatocytes and adipocytes was also increased in response to myocardial ischaemia/reperfusion injury [34]. FGF21 binds to its receptor complex in cardiomyocytes, protecting myocardial function by reducing caspase 3 activity, cell death, 
and myocardial infarction [34]. FGF21 also prevents atherosclerosis development in mice [35]. In atherosclerotic rodent models, FGF21 administration can ameliorate dyslipidemia and vascular atherosclerotic lesions, and reduce endoplasmic reticulum stress-mediated apoptosis [36], and reactive oxygen species production [37]. These findings suggested a protective role of FGF21 in cardiac hypertrophy, ischaemia/reperfusion injury in cardiac surgery and atherosclerosis, all of which are associated with AF development [38-40]. Therefore, based on the animal data, the role of FGF21 in relation to AF in humans deserves further study.

Although the present study did not demonstrate an association between baseline plasma FGF21 levels and incident AF, both FGF21 levels and AF are associated with a number of CVD risk factors. Current evidence supports the association between FGF21 levels and AF in patients without other severe comorbidities [9], and patients with rheumatic heart disease [10]. Furthermore, FGF21 might positively correlate with atrial fibrosis in AF patients with rheumatic heart disease [10]. However, there are some limitations in these studies. Subjects recruited in these studies were mainly Chinese and had a small sample size. The relationship of FGF21 levels with AF may differ in people with and without rheumatic heart disease. The cross-sectional study design also limits the assessment of a causal relationship between FGF21 levels and AF. Our study has the advantage of making use of data with good quality control as part of a large well-characterized sample of participants apparently free of clinical CVD at the time of recruitment. The longitudinal study design of the MESA study also allows the analysis of the temporal relationship between FGF21 levels and incident AF. The long follow-up period of 12.9 years is also another strength of the study. However, there are several limitations of our study. The major limitation is that FGF21 levels were assessed at baseline only. Therefore, longitudinal analysis of the change in FGF21 levels is not possible. Moreover, in this analysis, only circulating levels of FGF21 were measured, but not its local expression in atrial tissues or other heart tissues. We could not exclude the possibility that AF was under diagnosed in this study, especially for paroxysmal AF, as AF was not defined by frequent or extended electrocardiographic monitoring.

The literature suggests that circulating FGF21 levels may be a potential biomarker for AF that could be combined with other biomarkers to aid screening and risk stratification of incident AF. However, our findings suggest that this may not be the case as baseline FGF21 levels were not predictive of incident AF in our study. Further studies are needed to elucidate whether FGF21 has a role in AF development using other human populations, or animal studies.

\section{Supplementary Material}

Refer to Web version on PubMed Central for supplementary material.

\section{Acknowledgments}

The authors thank the other investigators, the staff, and the participants of the MESA study for their valuable contributions. A full list of participating MESA investigators and institutions can be found at http://www.mesanhlbi.org. 
Financial support: The FGF21 level measurement was supported by the NSW CVRN Research Development Project Grant (100715) from the National Heart Foundation of Australia to Dr. Kwok Leung Ong. Kwok Leung Ong was supported by the Vice-Chancellor's Postdoctoral Fellowship from the University of New South Wales (RG134592) and the Australian National Health and Medical Research Council Career Development Fellowship (1122854). The MESA study was supported by contracts HHSN268201500003I, N01-HC-95159, N01-HC-95160, N01-HC-95161, N01-HC-95162, N01-HC-95163, N01-HC-95164, N01-HC-95165, N01-HC-95166, N01HC-95167, N01-HC-95168, N01-HC-95169 and R01-HL-127659 from the National Heart, Lung, and Blood Institute, by grants UL1-TR-000040, UL1-TR-001079, and UL1-TR-001420 from National Center for Advancing Translational Sciences.

\section{References}

1. Schnabel RB, Yin X, Gona P, Larson MG, Beiser AS, McManus DD, et al. 50 year trends in atrial fibrillation prevalence, incidence, risk factors, and mortality in the Framingham Heart Study: a cohort study. Lancet. 2015; 386:154-162. [PubMed: 25960110]

2. Sanna T, Diener HC, Passman RS, Di Lazzaro V, Bernstein RA, Morillo CA, et al. Cryptogenic stroke and underlying atrial fibrillation. N Engl J Med. 2014; 370:2478-2486. [PubMed: 24963567]

3. Chang KW, Hsu JC, Toomu A, Fox S, Maisel AS. Clinical Applications of Biomarkers in Atrial Fibrillation. Am J Med. 2017; 130:1351-1357. [PubMed: 28822701]

4. Sinner MF, Stepas KA, Moser CB, Krijthe BP, Aspelund T, Sotoodehnia N, et al. B-type natriuretic peptide and $\mathrm{C}$-reactive protein in the prediction of atrial fibrillation risk: the CHARGE-AF Consortium of community-based cohort studies. Europace. 2014; 16:1426-1433. [PubMed: 25037055]

5. Kharitonenkov TL, Shiyanova A, Koester AM, Ford R, Micanovic EJ, Galbreath EJ, et al. FGF-21 as a novel metabolic regulator. J Clin Invest. 2005; 115:1627-1635. [PubMed: 15902306]

6. Badman MK, Pissios P, Kennedy AR, Koukos G, Flier JS, Maratos-Flier E, et al. Hepatic fibroblast growth factor 21 is regulated by PPARalpha and is a key mediator of hepatic lipid metabolism in ketotic states. Cell Metab. 2007; 5:426-437. [PubMed: 17550778]

7. Inagaki T, Dutchak P, Zhao G, Ding X, Gautron L, Parameswara V, et al. Endocrine regulation of the fasting response by PPARalpha-mediated induction of fibroblast growth factor 21. Cell Metab. 2007; 5:415-425. [PubMed: 17550777]

8. Woo YC, Xu A, Wang Y, Lam KS. Fibroblast growth factor 21 as an emerging metabolic regulator: clinical perspectives. Clin Endocrinol (Oxf). 2013; 78:489-496. [PubMed: 23134073]

9. Han X, Chen C, Cheng G, Xie C, Yang M, Shou X, et al. Serum fibroblast growth factor 21 levels are increased in atrial fibrillation patients. Cytokine. 2015; 73:176-180. [PubMed: 25766411]

10. Wang R, Yi X, Li X, Jiang X. Fibroblast growth factor-21 is positively associated with atrial fibrosis in atrial fibrillation patients with rheumatic heart disease. Int J Clin Exp Pathol. 2015; 8:14901-14908. [PubMed: 26823820]

11. Bild DE, Bluemke DA, Burke GL, Detrano R, Diez Roux AV, Folsom AR. Multi-Ethnic Study of Atherosclerosis: objectives and design. Am J Epidemiol. 2002; 156:871-881. [PubMed: 12397006]

12. Patton KK, Heckbert SR, Alonso A, Bahrami H, Lima JA, Burke G, et al. N-terminal pro-B-type natriuretic peptide as a predictor of incident atrial fibrillation in the Multi-Ethnic Study of Atherosclerosis: the effects of age, sex and ethnicity. Heart. 2013; 99:1832-1836. [PubMed: 24131775]

13. Maan A, Jorgensen NW, Mansour M, Dudley S, Jenny NS, Defilippi C, et al. Association between Heat Shock Protein-60 and Development of Atrial Fibrillation: Results from the Multi-Ethnic Study of Atherosclerosis (MESA). Pacing Clin Electrophysiol. 2016; 39:1373-1378. [PubMed: 27807875]

14. Ong KL, Rye KA, O'Connell R, Jenkins AJ, Brown C, Xu A, et al. Long-term fenofibrate therapy increases fibroblast growth factor 21 and retinol-binding protein 4 in subjects with type 2 diabetes. J Clin Endocrinol Metab. 2012; 97:4701-4708. [PubMed: 23144467]

15. Ong KL, Januszewski AS, O'Connell R, Jenkins AJ, Xu A, Sullivan DR, et al. The relationship of fibroblast growth factor 21 with cardiovascular outcome events in the Fenofibrate Intervention and Event Lowering in Diabetes study. Diabetologia. 2015; 58:464-473. [PubMed: 25425220] 
16. Veeranna V, Zalawadiya SK, Niraj A, Kumar A, Ference B, Afonso L. Association of novel biomarkers with future cardiovascular events is influenced by ethnicity: results from a multi-ethnic cohort. Int J Cardiol. 2013; 166:487-493. [PubMed: 22240756]

17. Wallace TM, Levy JC, Matthews DR. Use and abuse of HOMA modeling. Diabetes Care. 2004; 27:1487-1495. [PubMed: 15161807]

18. Levey AS, Stevens LA, Schmid CH, Zhang YL, Castro AF, Feldman HI, et al. A new equation to estimate glomerular filtration rate. Ann Intern Med. 2009; 150:604-612. [PubMed: 19414839]

19. Choi EY, Bahrami H, Wu CO, Greenland P, Cushman M, Daniels LB, et al. N-terminal pro-B-type natriuretic peptide, left ventricular mass, and incident heart failure: Multi-Ethnic Study of Atherosclerosis. Circ Heart Fail. 2012; 5:727-734. [PubMed: 23032197]

20. Seliger SL, Hong SN, Christenson RH, Kronmal R, Daniels LB, Lima JA, et al. High Sensitive Cardiac Troponin $\mathrm{T}$ as an Early Biochemical Signature for Clinical and Subclinical Heart Failure: The Multi-Ethnic Study of Atherosclerosis. Circulation. 2017; 135:1494-1505. [PubMed: 28159799]

21. Bertoni AG, Whitt-Glover MC, Chung H, Le KY, Barr RG, Mahesh M, et al. The association between physical activity and subclinical atherosclerosis: the Multi-Ethnic Study of Atherosclerosis. Am J Epidemiol. 2009; 169:444-454. [PubMed: 19075250]

22. Borrell LN, Diez Roux AV, Jacobs DR Jr, Shea S, Jackson SA, Shrager S, et al. Perceived racial/ ethnic discrimination, smoking and alcohol consumption in the Multi-Ethnic Study of Atherosclerosis (MESA). Prev Med. 2010; 51:307-312. [PubMed: 20609433]

23. Natori S, Lai S, Finn JP, Gomes AS, Hundley WG, Jerosch-Herold M, et al. Cardiovascular function in multi-ethnic study of atherosclerosis: normal values by age, sex, and ethnicity. Am J Roentgenol. 2006; 186:S357-S365. [PubMed: 16714609]

24. Shen Y, Ma X, Zhou J, Pan X, Hao Y, Zhou M, et al. Additive relationship between serum fibroblast growth factor 21 level and coronary artery disease. Cardiovasc Diabetol. 2013; 12:124. [PubMed: 23981342]

25. Chow WS, Xu A, Woo YC, Tso AW, Cheung SC, Fong CH, et al. Serum fibroblast growth factor-21 levels are associated with carotid atherosclerosis independent of established cardiovascular risk factors. Arterioscler Thromb Vasc Biol. 2013; 33:2454-2459. [PubMed: 23887638]

26. Chen C, Cheung BM, Tso AW, Wang Y, Law LS, Ong KL, et al. High plasma level of fibroblast growth factor 21 is an independent predictor of type 2 diabetes: a 5.4-year population-based prospective study in Chinese subjects. Diabetes Care. 2011; 34:2113-2115. [PubMed: 21750278]

27. Lee Y, Lim S, Hong ES, Kim JH, Moon MK, Chun EJ, et al. Serum FGF21 concentration is associated with hypertriglyceridaemia, hyperinsulinaemia and pericardial fat accumulation, independently of obesity, but not with current coronary artery status. Clin Endocrinol (Oxf). 2014; 80:57-64. [PubMed: 23278761]

28. Mraz M, Bartlova M, Lacinova Z, Michalsky D, Kasalicky M, Haluzikova D, et al. Serum concentrations and tissue expression of a novel endocrine regulator fibroblast growth factor-21 in patients with type 2 diabetes and obesity. Clin Endocrinol (Oxf). 2009; 71:369-375. [PubMed: 19702724]

29. Semba RD, Crasto C, Strait J, Sun K, Schaumberg DA, Ferrucci L, et al. Elevated serum fibroblast growth factor 21 is associated with hypertension in community-dwelling adults. J Hum Hypertens. 2013; 27:397-399. [PubMed: 23190795]

30. Lin Z, Zhou Z, Liu Y, Gong Q, Yan X, Xiao J, et al. Circulating FGF21 levels are progressively increased from the early to end stages of chronic kidney diseases and are associated with renal function in Chinese. PLoS One. 2011; 6:e18398. [PubMed: 21525989]

31. Planavila A, Redondo I, Hondares E, Vinciguerra M, Munts C, Iglesias R, et al. Fibroblast growth factor 21 protects against cardiac hypertrophy in mice. Nat Commun. 2013; 4:2019. [PubMed: 23771152]

32. Planavila A, Redondo-Angulo I, Ribas F, Garrabou G, Casademont J, Giralt M, et al. Fibroblast growth factor 21 protects the heart from oxidative stress. Cardiovasc Res. 2015; 106:19-31. [PubMed: 25538153] 
33. Cong WT, Ling J, Tian HS, Ling R, Wang Y, Huang BB, et al. Proteomic study on the protective mechanism of fibroblast growth factor 21 to ischemia-reperfusion injury. Can J Physiol Pharmacol. 2013; 91:973-984. [PubMed: 24117266]

34. Liu SQ, Roberts D, Kharitonenkov A, Zhang B, Hanson SM, Li YC, et al. Endocrine protection of ischemic myocardium by FGF21 from the liver and adipose tissue. Sci Rep. 2013; 3:2767. [PubMed: 24067542]

35. Lin Z, Pan X, Wu F, Ye D, Zhang Y, Wang Y, et al. Fibroblast growth factor 21 prevents atherosclerosis by suppression of hepatic sterol regulatory element-binding protein-2 and induction of adiponectin in mice. Circulation. 2015; 131:1861-1871. [PubMed: 25794851]

36. Wu X, Qi YF, Chang JR, Lu WW, Zhang JS, Wang SP, et al. Possible role of fibroblast growth factor 21 on atherosclerosis via amelioration of endoplasmic reticulum stress-mediated apoptosis in apoE(-/-) mice. Heart Vessels. 2015; 30:657-668. [PubMed: 25092223]

37. Zhu W, Wang C, Liu L, Li Y, Li X, Cai J, et al. Effects of fibroblast growth factor 21 on cell damage in vitro and atherosclerosis in vivo. Can J Physiol Pharmacol. 2014; 92:927-935. [PubMed: 25313624]

38. Chrispin J, Jain A, Soliman EZ, Guallar E, Alonso A, Heckbert SR, et al. Association of electrocardiographic and imaging surrogates of left ventricular hypertrophy with incident atrial fibrillation: MESA (Multi-Ethnic Study of Atherosclerosis). J Am Coll Cardiol. 2014; 63:20072013. [PubMed: 24657688]

39. Heeringa J, van der Kuip DA, Hofman A, Kors JA, van Rooij FJ, Lip GY, et al. Subclinical atherosclerosis and risk of atrial fibrillation: the Rotterdam study. Arch Intern Med. 2007; 167:382-387. [PubMed: 17325300]

40. Elahi MM, Flatman S, Matata BM. Tracing the origins of postoperative atrial fibrillation: the concept of oxidative stress-mediated myocardial injury phenomenon. Eur J Cardiovasc Prev Rehabil. 2008; 15:735-741. [PubMed: 19020458] 


\section{Highlights}

- Previous small studies suggested FGF21 as a biomarker for atrial fibrillation.

- $\quad$ This study examined the relationship between FGF21 and incident AF.

- $\quad$ Among the 5,729 participants, 778 participants developed incident AF.

- Baseline FGF21 was not independently associated with incident AF. 


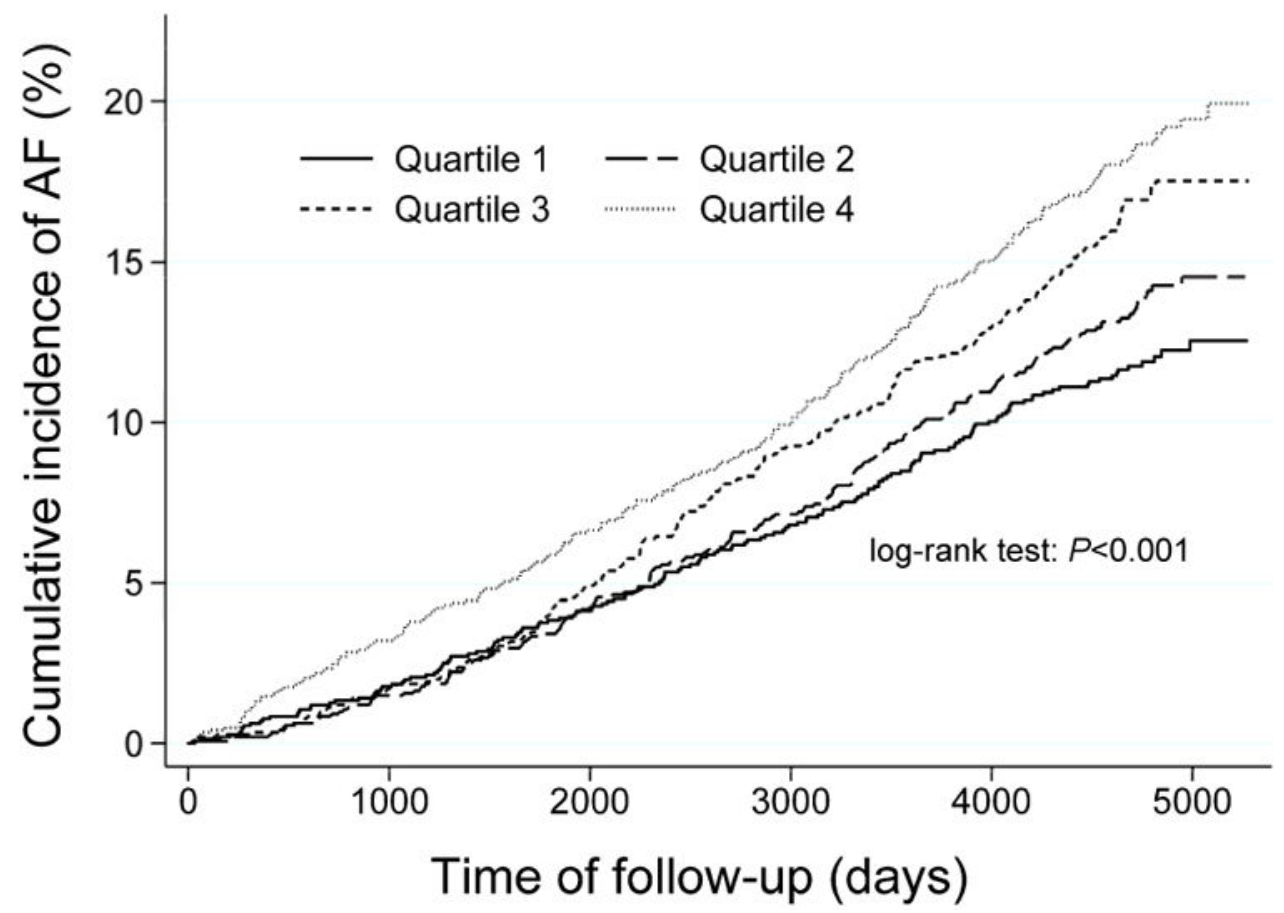

Fig. 1.

Kaplan-Meier curves for incident AF events across quartiles of FGF21 levels at baseline. 
Table 1

Baseline characteristics of participants with and without incident AF.

\begin{tabular}{|c|c|c|c|c|}
\hline \multirow[t]{2}{*}{ Characteristics } & \multirow[t]{2}{*}{$\mathbf{n}$} & \multicolumn{2}{|c|}{$\mathbf{A F}$} & \multirow[t]{2}{*}{$p \dagger$} \\
\hline & & Without $(n=4951)$ & With $(n=778)$ & \\
\hline \multicolumn{5}{|l|}{$\mathrm{n}$} \\
\hline Age, years & 5729 & $61.4(10.1)$ & $69.6(7.8)$ & $<0.001$ \\
\hline Women, \% & 5729 & $53.1(2628)$ & $45.4(353)$ & $<0.001$ \\
\hline Race/ethnicity, \% & - & - & - & $<0.001$ \\
\hline Caucasian & 2123 & $35.6(1761)$ & $46.5(362)$ & - \\
\hline African American & 1652 & $29.8(1477)$ & $22.5(175)$ & - \\
\hline Hispanic American & 1254 & $22.5(1113)$ & $18.1(141)$ & - \\
\hline Chinese American & 700 & $12.1(600)$ & $12.9(100)$ & - \\
\hline Education, $\%$ & - & - & - & 0.21 \\
\hline$<$ High school & 1046 & $18.0(886)$ & $20.6(160)$ & - \\
\hline High school & 2389 & $42.0(2073)$ & $40.7(316)$ & - \\
\hline$>$ High school & 2273 & $40.0(1973)$ & $38.7(300)$ & - \\
\hline No health insurance, $\%$ & 5709 & $9.4(466)$ & $5.4(42)$ & $<0.001$ \\
\hline Smoking, $\%$ & - & - & - & $<0.001$ \\
\hline Never & 2874 & $50.9(2511)$ & $46.8(363)$ & - \\
\hline Former & 2112 & $36.1(1779)$ & $42.9(333)$ & - \\
\hline Current & 723 & $13.0(643)$ & $10.3(80)$ & - \\
\hline Pack-years of smoking & 5647 & $10.5(19.6)$ & $15.6(25.4)$ & $<0.001$ \\
\hline Current alcohol use, $\%$ & - & - & - & 0.23 \\
\hline No or moderate consumption & 4075 & $71.4(3509)$ & $73.0(566)$ & - \\
\hline High consumption & 1075 & $18.8(927)$ & $19.1(148)$ & - \\
\hline Heavy drinking & 543 & $9.8(482)$ & $7.9(61)$ & - \\
\hline Physical activity, MET-hours/weeks & 5711 & $97.1(98.5)$ & $79.7(82.3)$ & $<0.001$ \\
\hline BMI, $\mathrm{kg} / \mathrm{m}^{2}$ & 5729 & $28.3(5.4)$ & $28.5(5.6)$ & 0.23 \\
\hline Waist-to-hip ratio & 5729 & $0.93(0.80)$ & $0.95(0.82)$ & $<0.001$ \\
\hline Height, cm & 5729 & $166.2(9.93)$ & $167.0(10.7)$ & 0.045 \\
\hline Heart rate, beats per minute & 5686 & $63.1(9.5)$ & $63.1(10.4)$ & 0.98 \\
\hline Diabetes, $\%$ & 5719 & $12.1(597)$ & $16.7(130)$ & $<0.001$ \\
\hline LDL cholesterol, mg/dL & 5651 & $117.7(31.5)$ & $114.0(31.2)$ & 0.003 \\
\hline HDL cholesterol, mg/dL & 5719 & $50.8(14.8)$ & $51.6(15.3)$ & 0.14 \\
\hline Triglycerides, $\mathrm{mg} / \mathrm{dL}^{a}$ & 5722 & $111.0(77.0-162.0)$ & $110.0(76.0-154.0)$ & 0.48 \\
\hline Lipid-lowering medication, $\%$ & 5717 & $15.9(783)$ & $22.4(174)$ & $<0.001$ \\
\hline Systolic BP, mmHg & 5727 & $125.8(21.2)$ & $134.3(22.2)$ & $<0.001$ \\
\hline Diastolic BP, mm Hg & 5727 & $72.0(10.3)$ & $72.4(10.3)$ & 0.25 \\
\hline Anti-hypertensive medication, $\%$ & 5727 & $35.2(1,740)$ & $52.1(405)$ & $<0.001$ \\
\hline Current aspirin use, $\%$ & 5494 & $18.4(871)$ & $30.1(226)$ & $<0.001$ \\
\hline HOMA2-IR ${ }^{a}$ & 5703 & $0.93(0.67-1.39)$ & $0.91(0.65-1.32)$ & 0.49 \\
\hline $\mathrm{eGFR}, \mathrm{mL} / \mathrm{min} / 1.73 \mathrm{~m}^{2}$ & 5719 & $79.0(16.1)$ & $71.5(17.1)$ & $<0.001$ \\
\hline
\end{tabular}




\begin{tabular}{lcccc}
\hline Characteristics & n & \multicolumn{2}{c}{ AF } & $p^{\dagger}$ \\
& & Without (n=4951) & With (n=778) & \\
\hline LV mass, g & 4166 & $144.3(38.7)$ & $153.7(44.0)$ & $<0.001$ \\
LV end-diastolic volume, mL & 4166 & $125.8(30.4)$ & $127.5(35.1)$ & 0.29 \\
LV ejection fraction, \% & 4166 & $69.0(7.2)$ & $69.0(8.60)$ & 0.97 \\
CRP, mg/L & 5696 & $1.90(0.83-4.22)$ & $1.91(0.90-4.08)$ & 0.35 \\
IL-6, pg/mL & 5586 & $1.19(0.77-1.88)$ & $1.42(0.91-2.17)$ & $<0.001$ \\
NT-proBNP, pg/mL & 5722 & $48.4(22.1-95.4)$ & $104.5(49.0-205.7)$ & $<0.001$ \\
FGF21 levels, pg/mL & 5729 & $142.8(78.5-239.5)$ & $166.0(93.9-283.0)$ & $<0.001$ \\
\hline
\end{tabular}

Statistics are shown as mean (SD), percent (n), or median (interquartile range). Comparison of clinical characteristics was performed by t-test for continuous variables and Chi-square test for categorical variables respectively.

${ }_{p}^{a}$ values were estimated using ln-transformed data. 


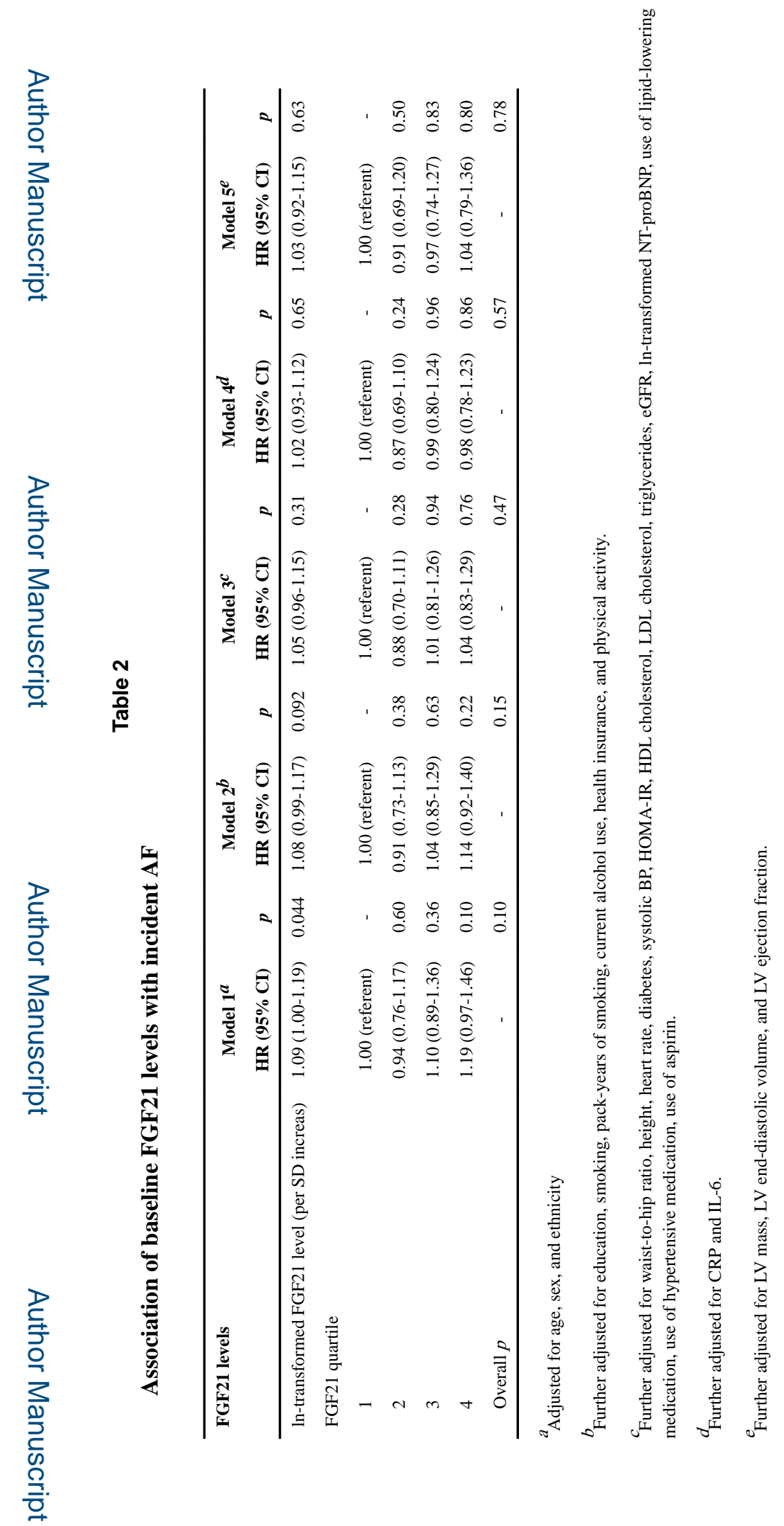

Atherosclerosis. Author manuscript; available in PMC 2019 February 01. 
Table 3

Association of baseline FGF21 levels with incident AF after further adjusting for incident CHD event

\begin{tabular}{lcc}
\hline FGF21 levels & HR $(\mathbf{9 5 \%}$ CI) & $\boldsymbol{p}$ \\
\hline ln-transformed FGF21 level (per SD increase) & $1.03(0.92-1.15)$ & 0.63 \\
FGF21 quartile & & \\
1 & 1.00 (referent) & - \\
2 & $0.90(0.68-1.18)$ & 0.44 \\
3 & $0.96(0.73-1.26)$ & 0.76 \\
4 & $1.03(0.78-1.35)$ & 0.85 \\
Overall $p$ & - & 0.77 \\
\hline
\end{tabular}

Data were adjusted for age, sex, ethnicity, education, smoking, pack-years of smoking, current alcohol use, health insurance, physical activity, waist-to-hip ratio, height, heart rate, diabetes, systolic BP, HOMA-IR, HDL cholesterol, LDL cholesterol, triglycerides, eGFR, In-transformed NTproBNP, use of lipid-lowering medication, use of hypertensive medication, use of aspirin, CRP, IL-6, LV mass, LV end-diastolic volume, LV ejection fraction, and incident CHD (as time-dependent variable). 